\section{RARE GENETIC MUTATION RELATED TO SEVERE NEUROMOTOR RETARDATION}

Aida Koka*. Vlora Pediatric Hospital

10.1136/archdischild-2021-europaediatrics.99

A 6 year old girl followed for severe mental and neuromotor retardation was examined for genetic abnormalities. The daughter of non-consanguinous parents, and sister of a normal brother, the child had important visual problems and significant facial dysmosrfism. Her cranial MRI showed mesial temporal sclerosis with hypocampal sulcii dilation, hypotrophy and cornual deformity. She had a cytogenomic microarray analysis performed. Her results showed a loss involving chromosome $1 \mathrm{p} 13.3 \mathrm{p} 13.2(7.32 \mathrm{Mb})$, indicating a deletion in this region, region that is held important for cases that were evaluated for neurological impairment,congenital anomalies and facial dysmorphism. The cytogenomic microarray analysis also showed a loss involving chromosome 2q23.3q24.1 (4.04 Mb), indicating a deletion in this region. A de novo deletion overlapping this region has been reported to be disease causing in one patient (Malbin J et al), a deletion that has been classified as Variant of Uncertain Significance.

RapidSureDeepDive is a cytogenetic method which detects copy number variants with a much higher resolution than conventional cytogenetic analysis. This technology, which does not require staining or cell culture, uses specially designed chips to study the DNA from various sample types.

The deletions found in our patient are quite rare, and maybe the variant of the second deletion will be reclassified as disease causing if proved to be denovo.

a 6 year old girl followed for severe mental and neuromotor retardation was examined for genetic abnormalities. the daughter of non-consanguinous parents, the child was found to have mesial temporal sclerosis with hypocmapal sulcii dilation.

RapidSureDeepDive is a cytogenetic method which detects copy number variants with a much higher resolution thanconventional cytogenetic analysis. This technology, which does not require staining or cell culture, uses specially designedchips to study the DNA from various sample types such as Blood, Amniotic Fluid, Tissue, Product of Conception etc. It isused to detect gains and losses of DNA throughout the human genome or for detection of loss of heterozygosity of SNPs The cytogenomic microarray analysis showed a loss involving chromosome

$1 \mathrm{p} 13.3 \mathrm{p} 13.2(7.32 \mathrm{Mb})$, indicating a deletion in this region. This deletion contains 85 OMIM genes. This deletion overlaps the region in patients submitted to the DECIPHER database: Patient ID: 383601,315409 and 331558: all these cases were evaluated for neurological impairment, congenital anomalies and facial dysmorphism. The submitted copy number variants have been classified as Likely pathogenic and hence the above variant has been classified as Likely Pathogenic. The cytogenomic microarray analysis also showed a loss involving chromosome $2 \mathrm{q} 23.3 \mathrm{q} 24.1(4.04 \mathrm{Mb})$, indicating a deletion in this region. This deletion contains 13 OMIM genes.

A de novo deletion overlapping this region has been reported to be disease causing in one patient (Malbin J et al).
The deleted region does not include MBD5, a gene commonly implicated in intellectual disability and hence the deletion has been classified as Variant of Uncertain Significance. The variant will be reclassified as disease causing if proved to be denovo.

\section{WILLIAMS-BEUREN SYNDROME: A CASE SERIES}

${ }^{1}$ Dorian Laslo*, ${ }^{2}$ Višnja Tomac, ${ }^{1,2}$ Silvija Pušelijć. ${ }^{1}$ Faculty of medicine Osijek, Josip Juraj Strossmayer University of Osijek, Croatia; ${ }^{2}$ University Hospital Centre Osijek, Department of Pediatrics, Croatia

\subsection{6/archdischild-2021-europaediatrics. 100}

Williams-Beuren syndrome (WBS) is developmental disorder caused by microdeletion of genes from chromosome 7. It is estimated in 1 of 10000 people. WBS could be autosomaldominant inherited, but usually, it is caused by de novo microdeletion of 26-28 genes on chromosome 7. Usually genes such as CLIP2, NCF1, ELN, GTF2I, GTF2IRD1 and LIMK1 are deleted. ELN gene is detected as a cause of supravalvular aortic stenosis, while absence of NCF1 gene is related to hypertension. Patients with WBS are characterized by cardiovascular diseases, facial dysmorphic features, intellectual disability, unique personality character and endocrine abnormalities.

Case Report We present the case of three patients, two girls at the ages of one and three years and a boy at the age of two years. All three patients have healthy parents and brothers and sisters without known chronic or genetic diseases. All three were presented with facial dysmorphic features which include broad nasal bridge, microretrognathia, large mouth, upper lip is thin while lower lip is thicker. All three patients are cognitively deficient and they have speech problems. The female patient at the age of three years also has hypertension, small teeth, larger neurocranium, atrial and ventricular (muscular) septal defect, gastroesophageal reflux disease (GERD), hypothonia and endocrine disorders; hypercalcemia, hypercalciuria, hypothyroidism and early puberty. The male patient also has prominent ears, palmar crease on left palm, pulmonary stenosis with mitral regurgitation and bilateral inguinal hernia. All three patients were suspective for WBS. Karyograms of all patients were normal, but FISH analysis performed with ELN primer for detection of WBS proved microdeletion on chromosome 7 in region 11 (7q11).

Conclusion WBS is rare disorder characterised by wide range of symptoms and signs. Clinical diagnostic criteria are available for WBS, but conformation of diagnosis requires detection of microdeletion on chromosome 7q11.2. Early recognition of specific patterns of the disorder, such as facial dysmorphic features together with heart abnormalities, cognitive deficiency and vision problems, is crucial because those patients need to take speech therapist and psychologist therapy as soon as possible because it improves their integration in social environment. Management is focused on treatment of symptoms (eg. hypertension, hypercalcemia), psychological and psychiatric evaluation and speech therapy. 